\title{
Corela
}

Cognition, représentation, langage

HS-25 | 2018

Les procédés implicites dans l'interface sémantiquepragmatique

\section{Les procédés implicites pris dans l'interface sémantique-pragmatique. Présentation}

Sophie Anquetil

\section{(2) OpenEdition}

Journals

Édition électronique

URL : http://journals.openedition.org/corela/5871

DOI : $10.4000 /$ corela. 5871

ISSN : 1638-573X

Éditeur

Cercle linguistique du Centre et de l'Ouest - CerLICO

Référence électronique

Sophie Anquetil, «Les procédés implicites pris dans l'interface sémantique-pragmatique. Présentation », Corela [En ligne], HS-25 | 2018, mis en ligne le 09 juillet 2018, consulté le 19 avril 2019. URL : http:// journals.openedition.org/corela/5871 ; DOI : 10.4000/corela.5871

Ce document a été généré automatiquement le 19 avril 2019

\section{cc) (†) (ㅇ)}

Corela - cognition, représentation, langage est mis à disposition selon les termes de la licence Creative Commons Attribution - Pas d'Utilisation Commerciale - Partage dans les Mêmes Conditions 4.0 International. 


\title{
Les procédés implicites pris dans l'interface sémantique- pragmatique. Présentation
}

\author{
Sophie Anquetil
}

1 Depuis Grice (1957), la pragmatique oppose le dire explicite au dire implicite. Cette opposition fondamentale entre ce qui est dit et ce qui est implicité dans un énoncé - ou entre aspects vériconditionnels et aspects non vériconditionnels des énoncés - a donné naissante à une pluralité de concepts : le présupposé, le sous-entendu, le trope illocutoire, l' implicitation, pour ne citer qu'eux. Cette variété d'objets nous confronte à la difficulté de représenter les strates de la signification impliquées par l'émergence d'un contenu implicite et à en proposer un mode de traitement qui tienne compte des particularités de chacun de ces objets. En effet, les présupposés, que l'on reconnaît parce qu'ils restent «affirmés par la négation de cet énoncé ou par sa transformation en question » (Ducrot, 1969 : 34), appartiennent « de plein droit au sens littéral ( ibid.: 35) et se situent « dans un passé de la connaissance, éventuellement fictif, auquel le locuteur fait semblant de se référer » (ibid. : 35). Les sous-entendus, au contraire, sont exclus de ce "sens littéral" et y apparaissent comme "surajoutés» (ibid. : 35), ce qui permet au locuteur "d'avancer quelque chose 'sans le dire, tout en le disant' " (ibid. : 35) et ainsi de se retrancher derrière ce "sens littéral". Le trope illocutoire renvoie, quant à lui, à une figure avec changement de sens, et consiste en un « renversement de la hiérarchie usuelle des niveaux sémantiques : sens littéral dégradé en contenu connoté, sens dérivé promu en contenu dénoté " "sous la pression de certains facteurs co(n)textuels » (Kerbrat-Orecchioni, 1986: 97). Il peut désigner un ensemble de figures : métaphore, métonymie, synecdoque, hyperbole, litote, antiphrase et ironie. Enfin, l'implicitation correspond chez Grice à ce qui est communiqué (vs. ce qui est dit) et comprend deux sous-classes : les implicitations conventionnelles et les implicitations non conventionnelles (Sadock 1978, Levinson 1983). Les premières sont déterminées uniquement à partir des mots et de la forme des phrases énoncées contrairement aux secondes qui se subdivisent en deux catégories: les implicitations conversationnelles et les implicitations non conversationnelles. Si les implicitations conversationnelles résultent d'une transgression du principe de coopération - lequel 
impose aux participants d'un échange de contribuer de manière rationnelle et coopérative pour faciliter l'interprétation des énoncés - et des maximes conversationnelles (quantité, qualité, relation, manière), les implicitations non conversationnelles sont, quant à elles, calculées sur la base du sens conventionnel des mots et des connaissances d'arrière-plan. Il faut encore distinguer parmi les implicitations conversationnelles, les implicitations généralisées et particulières. Ces deux catégories exploitent le principe de coopération et les maximes conversationnelles, mais elles se distinguent par le fait que les implicitations généralisées sont déclenchées uniquement à partir du matériau linguistique et sont indépendantes du contexte, ce qui n'est pas le cas des secondes.

2 Outre la complexité à représenter les différentes strates de la signification qui se superposent, la pluralité des objets associés à l'implicite brouille aussi la frontière qui sépare la sémantique de la pragmatique. En effet, si l'on considère que le champ d'investigation de la sémantique se limite aux aspects vériconditionnels des énoncés, alors les présupposés, sous-entendus, implicitations relèveront du champ de la pragmatique (Reboul et Moeschler, 1998). Si, en revanche, on envisage la sémantique comme l'étude des aspects conventionnels de la signification, il faudra faire appel aussi bien au domaine de la sémantique qu'au domaine de la pragmatique pour rendre compte des différentes facettes de la notion d'implicitation, selon qu'elle est de nature conventionnelle ou non.

3 Face à ces deux modes de traitement alternatifs, deux courants de la pragmatique moderne s'opposent, d'une part la pragmatique intégrée, de l'autre la pragmatique néogricéenne. Le premier, incarné par Anscombre et Ducrot (1983), «se refuse à (...) faire travailler [la pragmatique], après coup, sur les résultats de la description syntaxique et sémantique des énoncés "(Habert, 1982: 204) et la fait intervenir dès le niveau sémantique profond. Dans cette perspective, les mécanismes inférentiels, au cœur du processus interprétatif, sont de nature argumentative, et non pas déductive. Les aspects de l'énonciation étant inscrits dans le code, les contenus implicites peuvent être anticipés. Le lien inférentiel qui permet d'y avoir accès est intrinsèque à la langue.

Dans le cadre de la perspective néo-gricéenne, l'accès au contenu implicite ne se limite pas au strict décodage du matériau linguistique (modèle du code, Sperber et Wilson, 1989) ; il nécessite la mise en œuvre de mécanismes inférentiels de nature déductive (modèle inférentiel, ibid. 1989). Cette représentation du langage postule une hypothèse cognitive très forte sur le processus de compréhension des énoncés, dans la mesure où le processus interprétatif se scinde en deux étapes successives :

- la première s'attache à déchiffrer stricto sensu les éléments linguistiques de la phrase sous-jacente à l'énoncé (système linguistique) ;

- la seconde fait intervenir nos connaissances encyclopédiques sur le monde, les conditions d'énonciation ainsi que les principes conversationnels qui régissent la communication (système central).

5 Ainsi, cette représentation du processus interprétatif s'organise de façon séquentielle, et implique qu'un contenu implicite nécessite un effort cognitif plus important qu'un contenu explicite pour être interprété par l'interlocuteur.

6 La validité de ces hypothèses cognitives et interprétatives sur la nature du lien inférentiel serait donc dépendante de l'objet auquel on les soumet à l'analyse, ce qui nous incite à envisager un dispositif théorique capable de représenter la variété des processus 
interprétatifs mis en place pour accéder au sens implicite. Le numéro $\mathrm{n}^{\circ} 20$ de Corela, consacré à l'implicite (Elie-Deschamps, dir., 2016) s'est précisément attaché à engager une réflexion sur le traitement cognitif de l'implicite en proposant, à partir d'études de cas, des stratégies didactiques facilitant l'accès au sens implicite, que ce soit pour interpréter le discours humoristique (Charles), le débat polémique radiophonique (Wojciechowska \& Richard), des scénarii filmés (Laval, Gil et Hattouti), la dimension philosophique de l'image (Lerner et Kaheraoui), les signes vocaux (Rittaud-Huttinet), ou encore pour comprendre comment les processus de catégorisation lexicale interfèrent sur l'interprétation des formes implicites (Elie-Deschamps). Quelle que soit la stratégie didactique adoptée (guidage, étayage, analyser les mots, utiliser le contexte, inclure la prosodie signifiante, etc.), et quelle que soit la situation d'apprentissage explorée (classe de FLE, de $\mathrm{CP}$, de primaire, etc.), les résultats de ces études montrent que le concept d' implicite recouvre une pluralité d'objets (énoncés de type métaphorique, intentionnalité dite ludique, expressions idiomatiques, interjections, etc.) aux critères définitoires distincts, qui ont en commun de susciter une même activité cognitive, laquelle consiste à établir un lien inférentiel entre deux strates de la signification (Anquetil et Cozma, 2016). Force est de constater que la mise en relation entre ces deux strates de la signification est propre à tout processus interprétatif du fait de la plasticité du matériau linguistique, nous en venons à douter de la pertinence d'une délimitation franche entre implicite et explicite, voire même du concept d'implicite lui-même. Se posent alors les questions suivantes: l'implicite, bien que abondamment exploité par les chercheurs en pragmatique et analyse de discours, est-il réellement un concept opératoire ? Est-il un artefact de la linguistique ? Correspond-t-il réellement à une réalité cognitive, laquelle implique une organisation séquentielle en deux temps du traitement des énoncés? S'il existe, quels outils doivent être sollicités pour procéder au traitement linguistique des contenus implicites? Les outils exploités doivent-ils êtres issus de la sémantique ou de la pragmatique? Enfin, est-ce le concept d'implicite qui façonne les modèles théoriques ou les modèles théoriques qui façonnent le concept d'implicite?

7 Le présent numéro se propose donc de poursuivre la réflexion du numéro 20 dans une perspective épistémologique, en montrant que les objets d'étude qui fondent les courants de réflexion de la pragmatique contemporaine, leur mode de représentation des mécanismes de construction, mais aussi leurs dissensions théoriques, émanent de critères définitoires distincts. La réflexion épistémologique vise dans le même temps à proposer une approche théorique qui décloisonne délibérément les frontières entre la sémantique et de la pragmatique, voire même s'en affranchisse de façon à offrir un système de traitement commun aux différents objets issus du concept d'implicite.

8 La contribution d'olga Galatanu, qui ouvre ce numéro, s'attache à circonscrire cet objet que la sémantique argumentative nomme "implicite», qui diffère des contenus implicites problématisés par les théories contextualistes par le fait qu'il impose une reconstruction, dans l'interprétation du sens d'un énoncé ou d'un ensemble énoncés, d'un élément signifiant relevant de sa nature argumentative (Anscombre et Ducrot, 1983, Anscombre, éd., 1995). Ces implicites «argumentatifs» qui se situent « au niveau des conclusions autorisées par la signification lexicale» ou "d'une signification lexicale reconstruite discursivement ", se décrivent en termes d'enchainements discursifs à l'aide du modèle de la sémantique des possibles argumentatifs (SPA). L'intérêt de ce modèle sémantique pour le traitement des implicites argumentatifs est, d'une part, de rendre compte de mécanismes de transgression du protocole sémantique du mot par 
l'association d'un élément non conforme (non conformiste) à ce protocole, et d'autre part, d'envisager les probabilités de reconstruction discursive de la signification d'un mot absent dans le discours (Didier, 2015).

9 S'inscrivant lui aussi dans une perspective argumentative (la théorie des blocs sémantiques ou TBS), Kohei Kida interroge les compétences de la sémantique à décrire les phénomènes implicites. Cette interrogation peut surprendre de prime abord mais l'auteur justifie sa démarche en soulignant la nécessité de définir un objet qu'il situe du côté de la «pragmatique » - l'implicite - relativement à ses liens avec la sémantique, ou plutôt avec les sémantiques, référentielle d'une part, et argumentative d'autre part. Si l'objectif est de confronter les outils et modes de traitement offerts par ces deux courants de pensée, il est aussi surtout de développer le concept de "schéma argumentatif " introduit par la TBS et d'en montrer la pertinence pour le traitement des contenus implicites.

Dénonçant les «difficultés au développement d'une théorie sémantique systématique sans contexte, de même que l'aporie d'un contextualisme radical sans sémantique systématique », Jacques Mœschler souligne la nécessité d'une interface sémantiquepragmatique et la fragilité d'une frontière entre sémantique et pragmatique qui se trouve mise à mal avec l'existence des explicatures, de même que l'opposition entre sens explicite et sens implicite. L'auteur détermine quels critères définitoires sont susceptibles de distinguer les contenus sémantiques des contenus pragmatiques - l'enchaînement, la négation, ainsi que les connecteurs causaux parce que, donc, et - afin de mieux définir la nature des processus sémantiques et pragmatiques en jeu dans la construction du sens. L'intérêt de ces critères réside dans le fait qu'ils permettent de valider l'hypothèse cognitive selon laquelle les contenus implicites sémantiques sont moins accessibles que les contenus implicites pragmatiques.

11 Le rôle des principes d'inférence postulés par les théories néo-gricéennes dans la dérivation du sens est aussi au cœur de la recherche d'olivier Simonin. S'appuyant sur les travaux de Récanati (2004), l'implicature y est abordée comme un ensemble de processus pragmatiques déclenchés par l'application des maximes gricéennes. Mais contrairement à Récanati, l'auteur ne se conforme pas au modèle théorique gricéen ou néo-gricéen : il préfère recourir à l'appareil déductif de Sperber et Wilson (1989) pour dériver les implicatures, et montre l'intérêt de faire appel aux prémisses et conclusions d'un syllogisme sous-jacent pour reconstruire le sens.

12 L'objectif du travail d'Elodie Oursel est de proposer un modèle sémantique visant à dépasser l'opposition explicite-implicite, considérée comme inopérante, et dans le cadre duquel l'accès à l'intention de communication, qu'elle soit implicite ou non, ne repose pas uniquement sur les capacités d'inférence de l'interlocuteur, mais constitue plutôt une opération pluriactantielle, puisque l'intercompréhension repose sur la négociation du sens. En s'appuyant sur l'analyse des réactions issues d'un corpus de 72 conversations entre des agents administratifs français et des usagers étrangers, l'auteure met en lumière trois dimensions au cœur de cette négociation :

- une dimension situante,

- une dimension structurante,

- une dimension relationnelle.

13 Ainsi ce modèle se veut résoudre les problèmes de frontières, et ainsi de traitement de l'implicite, puisque les outils mobilisés ne scindent pas les contenus sémantiques et pragmatiques en deux groupes et transcendent ces deux champs disciplinaires. 

critères définitoires caractéristiques des différentes formes d'implicite - critères que toute tentative de modélisation doit prendre en compte pour être opératoire - propose un mode de traitement de l'implicite fondé sur une distinction entre niveau du contenu (implicitation du contenu énonciatif) et niveau de la responsabilité (implicitation de la responsabilité énonciative). L'originalité de ce mode de traitement réside dans le fait qu'il tend à mesurer l'intensité d'implicitation, autrement dit « la mesure dans laquelle chaque construction linguistique rend implicite la responsabilité du locuteur ou le contenu qu'il transmet » (Lombardi Vallauri \& Masia 2014), au moyen d'un système d'indices. Ainsi l'on parvient à quantifier l'implicitation du contenu et de la responsabilité, ici dans quelques textes de propagande politique (et commerciale), mais la méthode présente l'avantage de pouvoir être exploitée pour d'autres types de texte.

\section{BIBLIOGRAPHIE}

ANQUETIL S. (2013), Représentation et traitement des actes de langage indirects, Domaines

Linguistiques sous la direction de F. Neveu n 3, Série Formes discursives n 2, Classiques Garnier.

ANQUETIL S. \& COZMA A-M. (2016), « L'implicite : entre préconstruits sémantiques et détermination générique. Introduction ", Signes, discours et sociétés, $\mathrm{n}^{\circ} 17:$ http://revuesignes.gsu.edu.tr/?revue $=6$

ANSCOMBRE J-C. \& DUCROT O. (1983), L'argumentation dans la langue, Liège, Paris, Mardaga.

ANSCOMBRE J-C. (éd.) (1995), Théorie des topoï, Paris, Editions Kimé.

DIDIER S. (2015), La transmission de l'héritage culturel intangible par la littérature de jeunesse. Modélisation sémantico-discursive de «chez soi » dans le conte Mèyèno (Ponga, 2004), 20 mai 2015, cotutelle Université de Nantes, Macquarie University, Sydney, Australie, soutenue à l'Université de Nantes.

DUCROT O. (1969), « Présupposés et sous-entendus », Langue française, vol. 4, n 1, p. 30-43.

ELIE-DESCHAMPS J. (2016), « L’implicite, études de cas en linguistique appliquée », CORELA, HS-n - 20 : http://corela.revues.org/4659 ; DOI : 10.4000/corela.4659

GRICE H. P. (1957), « Meaning », The Philosophical Review, n 66, p. 377-388.

HABERT B. (1982), « Énonciation et argumentation : Oswald Ducrot », Mots, $n^{\circ}$ 5, en hommage à Robert-Léon Wagner, p. 203-218.

KERBRAT-ORECCHIONI C. (1986) L'implicite, Paris, Armand Colin.

LEVINSON S. C. (1983), Pragmatics, Cambridge, London, New York, Cambridge University Press.

LOMBARDI VALLAURI E. \& MASIA V. (2014), Implicitness Impact : measuring texts, Journal of Pragmatics, $\mathrm{n}^{\circ}$ 61, p. 161-184.

REBOUL A. \& MOESCHLER J. (1998), La pragmatique aujourd'hui, Paris, Seuil.

RECANATI F. (2004), Literal Meaning, Cambridge, Cambridge University Press. 
SADOCK J. M. (1978), « On testing for conversational implicature », in COLE P., Syntax and Semantics, vol. 9, Academic Press, New York, San Francisco, London, p. 281-297.

SPERBER D. \& WILSON D. (1989), La pertinence, Paris, Minuit.

\section{AUTEUR}

SOPHIE ANQUETIL

CeReS, EA 3648, Université de Limoges 